\title{
Triglyceride Reduces Cholesteryl Ester Accumulation and Maintains Lysosomal Acidification in Macrophage Foam Cells
}

\author{
Jody C. Ullery*, Brian E. Cox*, W. Gray Jerome* \\ * Vanderbilt University Medical Center, Department of Pathology, CC3321A MCN, Nashville, \\ TN 37232
}

Atherosclerosis is characterized by the presence of cholesterol-engorged foam cells that are derived from macrophages in the arterial intima. Cholesterol accumulation occurs through the uptake of a variety of lipid-containing particles. Internalized cholesterol accumulates within macrophage foam cells and such accumulations are primarily localized to lysosomes. While the majority of studies examining atherogenesis have focused on the role of cholesterol in the formation of macrophage foam cells, TG-rich lipoproteins, such as VLDL, are also present within the atherosclerotic lesion and are internalized by macrophages. Little is known about how this uptake might affect cholesterol metabolism and, specifically, the formation of cholesterol-rich macrophage foam cells. Therefore, our studies explore the effect of TG-rich lipoprotein particles, such as VLDL, on intracellular cholesterol metabolism. Results show that TG, delivered to the cell as a component of VLDL or TG-rich lipid dispersions, reduces cholesteryl ester (CE) accumulation in THP-1 macrophage foam cells. Reduced CE accumulation occurs in response to increased TG levels within the cell. Additionally, the accumulation of both TG and CE occurs within lysosomes. Triglyceride, delivered to the cell as a component of VLDL and TG-rich lipid dispersions, decreases the volume of lysosomes. Cholesterol accumulation in lysosomes inhibits acidification of lysosomes but lysosomal TG reduces this inhibition. Lipids, including TGs and cholesterol, are able to change the physical properties of the cell by changing the fluidity of cellular membranes and, potentially, lipid filled lysosomes. Therefore, one possible influence of TG-rich lipoproteins on cholesterol metabolism could be maintaining lysosomal cholesterol and cellular membranes in a more fluid state facilitating CE metabolism and cholesterol mobilization for efflux. The results of our studies have significance in identifying the role of TGs in foam cell development in regard to atherogenesis. 